\title{
Die meistzitierten Arbeiten im 35. Jahrgang der Psychiatrischen Praxis - Herzlichen Glückwunsch!
}

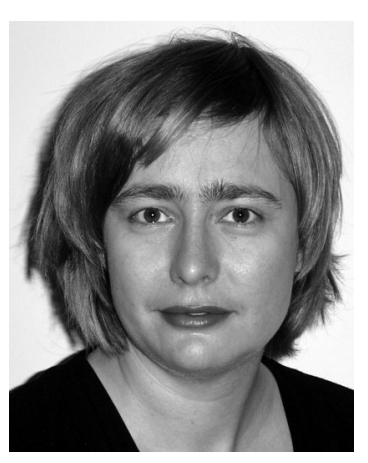

Dr. Heide Glaesmer

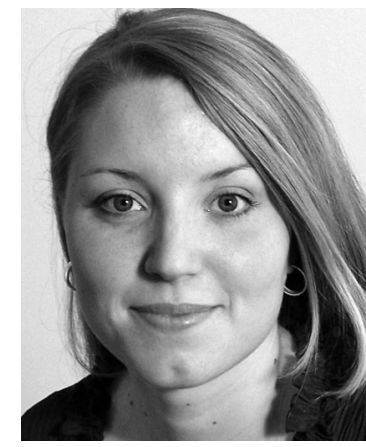

Dr. Tatjana Reichhart

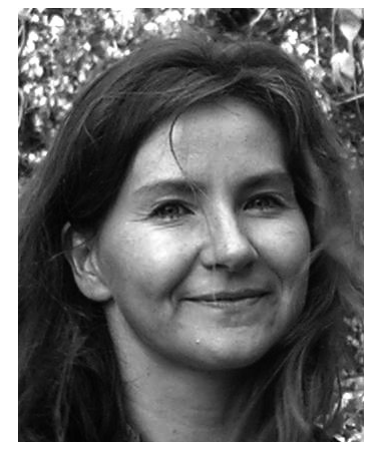

Dr. Christiane Roick
Auch in diesem Jahr küren wir wieder die meistzitierten Arbeiten der Psychiatrischen Praxis. Sie greifen brennende Themen der psychiatrischen Versorgung auf: Die Versorgung älterer Menschen, die Patientenbeteiligung in der Psychiatrie und neue Finanzierungsmodelle der Versorgung. Auf dem ersten Platz landete die Arbeit von Heide Glaesmer. Heide Glaesmer ist von Haus aus Psychologin, ist Gesundheitswissenschaftlerin und arbeitet gegenwärtig in der Selbstständigen Abteilung für Medizinische Psychologie und Medizinische Soziologie an der Universität Leipzig. Sie berichtet in ihrer Arbeit Ergebnisse einer bundesrepräsentativen Stichprobe von über 50-Jährigen. Sie konnte zeigen, dass psychische Beschwerden mit einem erhöhten Inanspruchnahme- und veränderten Krankheitsverhalten einher gehen. Dabei wird v.a. die primärärztliche Versorgung in Anspruch genommen [1].

Tatjana Reichhart, von Haus aus Ärztin und derzeit in einer Spezialambulanz für schizophren erkrankte Patienten am Zentrum für Disease Management, Klinik für Psychiatrie und Psychotherapie der TU München tätig, landete mit ihrer Übersichtsarbeit zur Patientenbeteiligung in der Psychiatrie auf Platz 2. In ihrer Arbeit konnte sie aufzeigen, dass die meisten psychiatrischen Patienten eine Beteiligung wünschen. Bei der Umsetzung in die Praxis ergibt sich jedoch noch ein großer Spielraum für Verbesserungen [2]. Die Arbeit von Frau Reichhart regte intensive Diskussionen in der Psychiatrischen Praxis an und zu dieser Arbeit wurde auch ein Kommentar von Martin Härter, jetzt Universitätsklinikum Hamburg-Eppendorf veröffentlicht [2].

Schon seit geraumer Zeit wird darüber nachgedacht, wie mit neuen Finanzierungsmodellen und veränderten Anreizstrukturen für die Leistungsanbieter eine effizientere Versorgung unserer Patienten möglich werden kann. Christiane Roick, Medizinerin und Gesundheitswissenschaftlerin, legte eine Analyse zu den Auswirkungen eines re- gionalen Psychiatriebudgets vor. Die Arbeit entstand in der Arbeitsgruppe von Hans-Helmut König, früher Leipzig, jetzt Hamburg-Eppendorf. Frau Roick konnte zeigen, dass die stationären Kosten in der Modellregion sanken, bei gleichzeitiger Intensivierung der intsitutsambulanten und teilstationären Versorgung. Im gleichen Zuge verbesserte sich das Funktionsniveau der Patienten in der Modellregion, sodass regionale Psychiatriebudgets die Kosten stationärer Versorgung reduzieren können, ohne dass es zu Einschränkungen in der Versorgungsqualität kommt [3].

Wir freuen uns für die Preisträgerinnen! Die aufgegriffenen Themen werden auch weiterhin in der Psychiatrischen Praxis diskutiert [4-6]. Wir gratulieren ganz herzlich und wünschen den Preisträgerinnen alles Gute!

\section{Steffi Riedel-Heller}

\section{Literatur}

1 Glaesmer H, Gunzelmann T, Martin A et al. Die Bedeutung psychischer Beschwerden für die medizinische Inanspruchnahme und Krankheitsverhalten Älterer. Psychiat Prax 2008; 35: 187-193

2 Reichhart T, Kissling W, Scheuring E et al. Patientenbeteiligung in der Psychiatrie - eine kritische Bestandsaufnahme. Psychiat Prax 2008; 35: 111-121

3 Roick C, Heinrich S, Deister A et al. Das Regionale Psychiatriebudget: Kosten und Effekte eines neuen sektorübergreifenden Finanzierungsmodells für die psychiatrische Versorgung. Psychiat Prax 2008; 35: 279285

4 Donath C, Bleich S, Gräßel E. Gerontopsychiatrische Tageskliniken - Prädiktoren der Inanspruchnahme und Qualitätserwartungen aus Sicht pflegender Angehöriger von Demenzpatienten. Psychiat Prax 2009; 36: 175-181

5 Borbé R, Jaeger S, Steinert T. Behandlungsvereinbarungen in der Psychiatrie. Psychiat Prax 2009; 36: 7-15

6 König H-H, Heinrich S, Heider D et al. Das Regionale Psychiatriebudget (RPB): Ein Modell für das neue pauschalierende Entgeltsystem psychiatrischer Krankenhausleistungen? Analyse der Kosten und Effekte des RPB nach 3,5 Jahren Laufzeit. Psychiat Prax 2010; 37: 34-42 